\title{
Vertical flows and structures excited by magnetic activity in the Galactic center region
}

\author{
Kensuke Kakiuchi ${ }^{1}$, Takeru K. Suzuki ${ }^{1,2}$, Yasuo Fukui ${ }^{1}$, \\ Kazufumi Torii ${ }^{1,3}$, Mami Machida ${ }^{4}$ and Ryoji Matsumoto ${ }^{5}$ \\ ${ }^{1}$ Dept. of Physics, Nagoya University, Furo-cho, Chikusa, Nagoya, Aichi, 464-8602, Japan \\ email: kakiuchi@nagoya-u.jp \\ ${ }^{2}$ School of Arts \& Sciences, The University of Tokyo, \\ 3-8-1, Komaba, Meguro, Tokyo, 153-8902, Japan \\ ${ }^{3}$ National Radio Observatory, National Astronomical Observatory of Japan, 462-2, Nobeyama, \\ Minamimaki, Minamisaku, Nagano, 384-1305, Japan \\ ${ }^{4}$ Dept. of Physics, Faculty of Sciences, Kyushu University, 6-10-1 Hakozaki, Higashi-ku, \\ Fukuoka 812-8581, Japan \\ ${ }^{5}$ Dept. of Physics, Graduate School of Science, Chiba University, 1-33 Yayoi-cho, Inage-ku, \\ Chiba 263-8522, Japan
}

\begin{abstract}
Various observations show peculiar features in the Galactic Center region, such as loops and filamentary structure. It is still unclear how such characteristic features are formed. Magnetic field is believed to play very important roles in the dynamics of gas in the Galaxy Center. Suzuki et al.(2015) performed a global magneto-hydrodynamical simulation focusing on the Galactic Center with an axisymmetric gravitational potential and claimed that non-radial motion is excited by magnetic activity. We further analyzed their simulation data and found that vertical motion is also excited by magnetic activity. In particular, fast down flows with speed of $\sim 100 \mathrm{~km} / \mathrm{s}$ are triggered near the footpoint of magnetic loops that are buoyantly risen by Parker instability. These downward flows are accelerated by the vertical component of the gravity, falling along inclined field lines. As a result, the azimuthal and radial components of the velocity are also excited, which are observed as high velocity features in a simulated positionvelocity diagram. Depending on the viewing angle, these fast flows will show a huge variety of characteristic features in the position-velocity diagram.
\end{abstract}

Keywords. accretion, accretion discs, Galaxy: bulge, Galaxy: centre - Galaxy: kinematics and dynamics, magnetohydrodynamics (MHD) - turbulence

We analyzed flows and magnetic fields obtained from the MHD simulation by Suzuki et al. (2015). Figure 1 shows trajectories of fluid elements from $t=399.5$ to $405.5 \mathrm{Myr}$ located in 0.3-0.4 kpc. These fluid elements rotate in the clockwise direction with speed $\approx 200 \mathrm{~km} \mathrm{~s}^{-1}$. In addition to the rotation, some fluid elements show fast vertical and/or radial motion with speed $\sim 10-100 \mathrm{~km} \mathrm{~s}^{-1}$. In Figure 2 we present the velocity and magnetic fields near a fast-downflow region. The downflow is accelerated by the vertical component of the gravity along the inclined field lines, which we call a magnetic sliding slope. The magnetic sliding slope is a part of a magnetic loop that is buoyantly lifted up by Paker instability (Parker 1966). Complex loop structures are observed near the Galactic centre (Fukui et al. 2006; Machida et al. 2009; Torii et al. 2010a). Our result indicates that downflows can be associated with these loops as discussed in Torii et al. (2010b). 


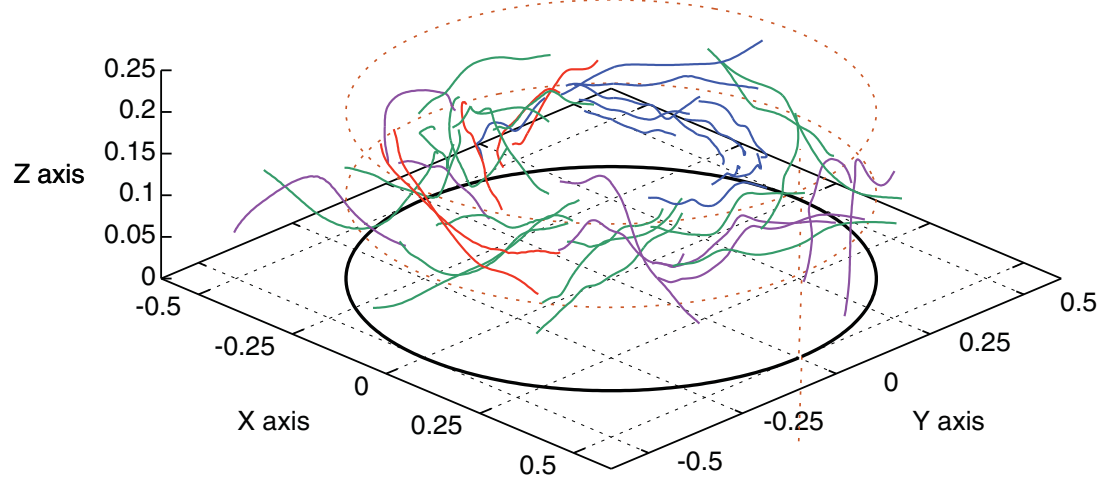

Figure 1. Trajectories of fluid elements from $t=399.5$ to $405.5 \mathrm{Myr}$. The unit of the axes is kpc. Flow patterns are classified by colours, red (monotonic upflows) green (monotonic downflows), purple (combination of upflows and downflows), and blue (no apparent up/downflow).

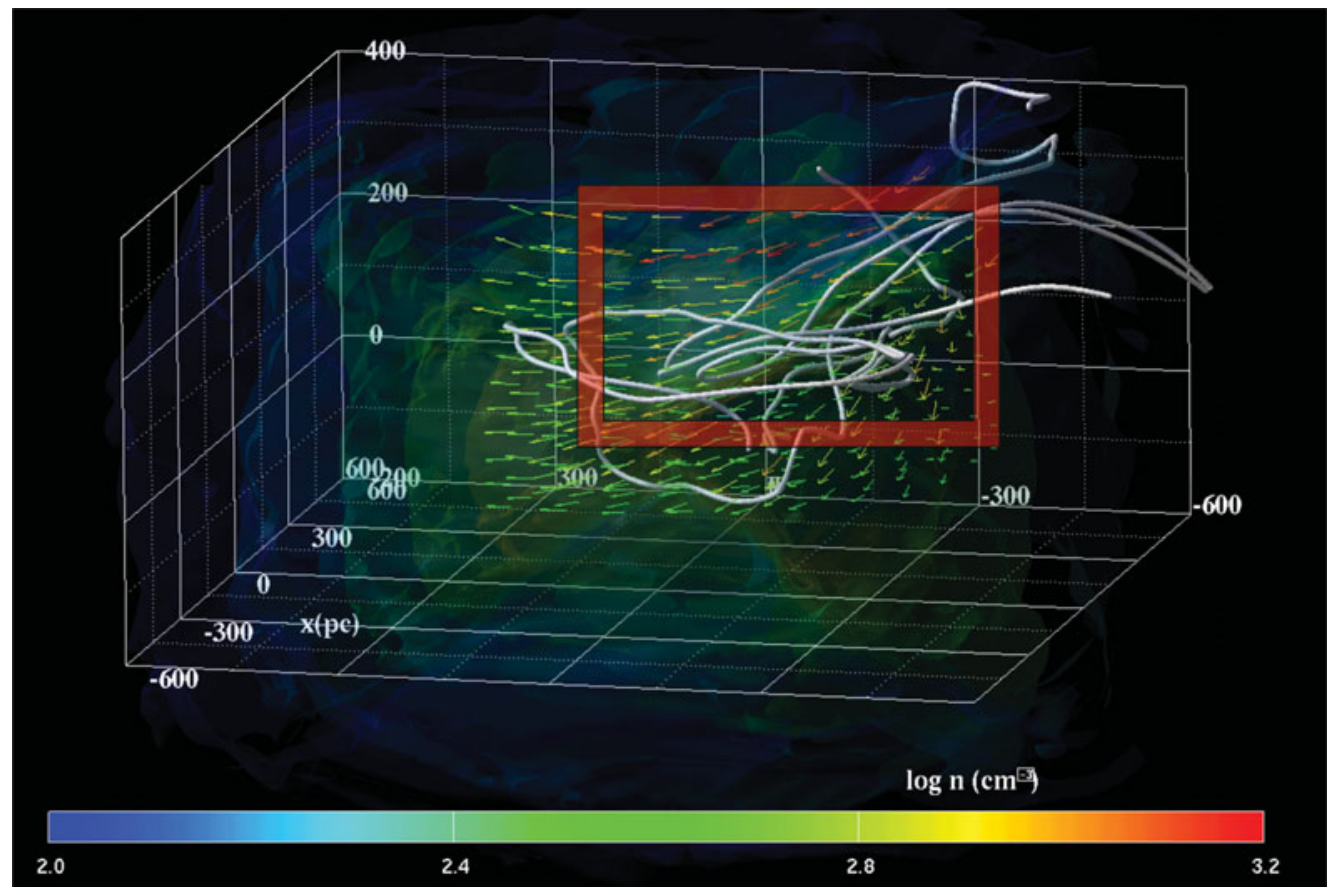

Figure 2. Magnetic field lines (white lines) and velocity field (arrows) near a fast-downflow region.

\section{References}

Fukui, Y., Yamamoto, H., Fujishita, M., et al. 2006, Science, 314, 106

Machida, M., Matsumoto, R., Nozawa, S., et al. 2009, PASJ, 61, 411

Parker, E. N. 1966, ApJ, 145, 811

Suzuki, T. K., Fukui, Y., Torii, K., Machida, M., \& Matsumoto, R. 2015, MNRAS, 454, 3049

Torii, K., Kudo, N., Fujishita, M., et al. 2010a, PASJ, 62, 1307

—. 2010b, PASJ, 62, 675 